\title{
MOTIVATED BEHAVIOR AND THE ESTROUS CYCLE IN RATS
}

\author{
Meir Steiner*, Richard J. Katz, Giulio Baldrighi and Bernard J. Carroll \\ Mental Health Research Institute, University of Michigan, Ann Arbor, MI 48109, U.S.A.
}

(Received 1 October 1979; in revised form 26 June 1980)

\begin{abstract}
SUMMARY
(1) The estrous cycle in the rat may be used to study recurrent changes in motor behaviors and motivation which are strongly related to cyclic hormonal and CNS changes. (2) The peak in motivated behaviors occurs during a sharply defined period on the night between proestrus and estrus and is evident in facilitated wheel-running, lordosis, and intracranial self-stimulation. (3) Behaviors without a clearly motivated character do not show an estrous cyclicity. (4) The estrous cyclic variation in intracranial self-stimulation was observed at a specific locus - the pars campacta of the substantia nigra. (5) A neurochemical link between sexually motivated behavior, wheel running and intracranial self-stimulation is suggested. This link is in part dopaminergic but is probably also activated by many other systems.
\end{abstract}

Key Words-Estrous cycle; wheel running; intracranial self-stimulation; motivated behavior.

\section{INTRODUCTION}

TWO BEHAVIORS, namely sexual receptivity (expressed as lordosis) and wheel-running (WR), are known to increase significantly on the night between proestrus and estrus in female cycling rats (Wang, 1923). Sexual receptivity, i.e. the intensity of the copulatory act or the readiness to respond to mating attempts of another individual, cannot be taken as an exclusive measure of sexually motivated behavior, however. Rather, the seeking of sexual contact must be considered along with the consummatory act itself. Utilizing different experimental techniques (Warner, 1927; Meyerson \& Lindstrom, 1973), it has been established that sexually motivated behavior, i.e. solicitation, also increases in the cycling female rat on the night between proestrus and estrus. This may point to a more general alteration in motivation during the estrous cycle. Activity levels, particularly those associated with wheel running also have often been used to imply the presence of a more general estrus-related change in motivation (Baumeister, Hawkins \& Cromwell, 1964). Direct evidence for motivational properties of wheel running (WR) comes mainly from two sources. (a) Rats will press a lever to obtain access to WR (Kagan \& Berkun, 1954). It has also been shown (Livesey, Egger \& Meyer, 1972) that WR, in male rats, serves as a consummatory response and produces the same learning pattern (in a T-maze) as other drives. (b) Positively reinforcing lateral hypothalamic stimulation, in female rats, may elicit WR (Rosenquist \& Hoebel, 1968). Although the seeking of sexual contact and increased WR on the night between proestrus and estrus may be regarded as motivated or goal-oriented behaviors, the generality of other estrus-related changes in behavior and their relation to motivation is not yet fully determined.

Analysis of behaviors such as sleeping, grooming, rearing, locomotion, etc. failed to indicate any correlation between activity and estrous cyclicity (Bolles, 1963). This suggests

\footnotetext{
*Present address: Shalvata Psychiatric Center, Tel Aviv University Medical School, Hod Hasharon, POB 94,
} Israel. 
some degree of specificity to the behavioral changes in estrus. In a more recent study, Finger (1969) demonstrated that more than half the direct observations (including locomotion, cage biting, and exploration) were increased during estrus. A photoelectric recording score yielded a peak at estrus but only in half the cycles observed. These data provide no basis for deciding whether any measure of activity other than WR can be regarded as a useful dependent variable of estrous cyclicity. A further means of approaching estrus-related changes in motivation involves studies of intracranial self-stimulation (ICS). It has been suggested that ICS of certain areas of the hypothalamus in ovariectomized rats can be influenced by estrogen treatment (Meyerson, Wilkins \& Sawyer, 1969). In one experiment, however, the hormonal induction of estrous behavior in ovariectomized rats had no effect on septal self-stimulation (Hodos \& Valenstein, 1960). Some data indicate that the performance of intact regularly cycling female rats in pressing a bar for electrical stimulation of the hypothalamus changes during the estrous cycle (Prescott, 1966; Scott \& Hoebel, 1966; Moss, 1968). Others were unable to confirm such a relationship (Hitt \& Gerall. 1969; Lenzer, 1971: Drewett \& Herberg, 1975).

In the present study the estrous cycle of the rat was used to study further some of the underlying mechanisms of motivated behaviors. In particular a variety of behaviors with established motivational character, including ICS, was compared with other behaviors with a motivationally neutral character for estrus-related changes. We tested the hypotheses that estrus-related changes were specific to behaviors with a clear goal-directed character, i.e. to motivated behaviors.

\section{METHODS}

\section{Animals}

Aduit experimentally naive female albino rats (Holtzman, Wisconsin), 200-250g body weight, were housed under controlled conditions of temperature $\left(21^{\circ} \mathrm{C}\right.$ ) and illumination (lights on $0545-1815 \mathrm{hr}$ ). This photo period was chosen to be consistent with the study of Butcher, Collins \& Fugo (1974). Teklad $4 \%$ fat rodent diet and tap water were supplied ad libitum. Estrous cycles were followed by daily vaginal smears obtained between $0900 \mathrm{and} 1100 \mathrm{hr}$ by lavage (using the saline medicine-dropper technique) with "diestrus day l" defined as the first day in which leukocytes dominated the smear (Zarrow. Yochim \& McCarthy, 1964). The females were initially given an adjustment period of 14 days in their cages. Two additional consecutively regular 4-day estrous cycles were monitored prior to the experimental recording.

Apparatus and procedure

Activity wheels. Ten rats were individually housed in $36 \mathrm{~cm}$ diameter activity wheels (Lafayette Instrument $\left(C_{0}\right)$.. Indiana). A Veeder recording counter was connected to the drum to count revolutions in either direction. Sensing contacts (microswitches) attached to the hub registered the revolutions of the drum. Activity was recorded continuously on an hourly basis for one week. In addition, recordings were reduced to 3-hourly rates and to total $24-$ hr rates (midnight to midnight) of running for each animal.

Behavioral Taxonomy. The physical arrangements for this experiment were designed to permit clear observation of the rats at any point in the light-dark cycle, while reducing potential sources of distraction. The rats were individually housed in standard transparent Plexiglas cages $(40 \times 25 \times 20 \mathrm{~cm})$ in which they lived for about 3 weeks before the observations were started. Ten animals were observed for $3 \mathrm{~min}$ twice daily (between $0930-1030 \mathrm{hr}$ daylight and between $2130-2230 \mathrm{hr}$-dim red light) over the course of 3 estrous cycles. The categories of behavior used were: eating, drinking, locomotion, chewing, sniffing, 'Straub-tail', rearing, grooming, posturing, headbobbing, yawning, sleeping, and darting. Each category of behavior was scored as "not present" (0), "minimal" (1), "some" (2), and "most of the time" (3) according to its relative occurrence during the 3 min period. For analysis, only those categories of behavior which occurred with a mean frequency of at least 3 times per cycle will be considered. Totals for the less frequently performed behavioral categories were too small for the sampling method to provide reliable data.

The data were analyzed by means of the BMD08V-ANOVA program (Dixon, 1968; revised and adapted by the Statistical Research Laboratory. The University of Michigan, 1975). In addition to analysis of variance, spectral 
density analysis was used for the assessment of time series. Spectral analysis is essentially a means of fitting activity rhythms to sine curves, with relative spectral density serving to indicate relative proportional contributions to the overall variance of a time series. The particular tests of time series employed tested the presence of an underlying non-homogeneity of spectral density against a null hypothesis of equal spectral densities from all rhythmic processes.

Tilt cages. Ten rats were individually housed in $32 \times 25 \times 20 \mathrm{~cm}$ transparent Plexiglas tilt cages in which they lived for about 2 weeks before the experiment was started. The cages tilted on a central fulcrum as the rat moved from one part of the cage to the other. Activity in the tilt cages was recorded on an hourly basis, 24 hr per day for 28 consecutive days (seven 4-day estrous cycles for each animal). Daily scores were analyzed with tests for time series analysis using spectral density analysis and evaluated with Pyke's Kolmogorov-Smirnov (K-S) statistic (Fox \& Guire, 1976). Recordings were collated (programmed in BASIC) to 3-hourly rates and to total 24 hr rates of activity for each animal.

Intracranial self-stimulation. Standard $25 \times 18 \times 17 \mathrm{~cm}$ stainless steel cages were modified to allow chronic selfstimulation. The Wolf-DiCara-Simpson design was utilized (Wolf, DiCara \& Simpson, 1973). The wire mesh floor served as a stimulation ground and a hinged $14 \times 16 \mathrm{~cm}$ stainless steel plate mounted overhead served as a contact for delivery of current. Upward displacement of the overhead panel allowed circuit completion and stimulation delivery through a mounted brushing on the animal's head (Fig. 1). This design allowed continuous access to the

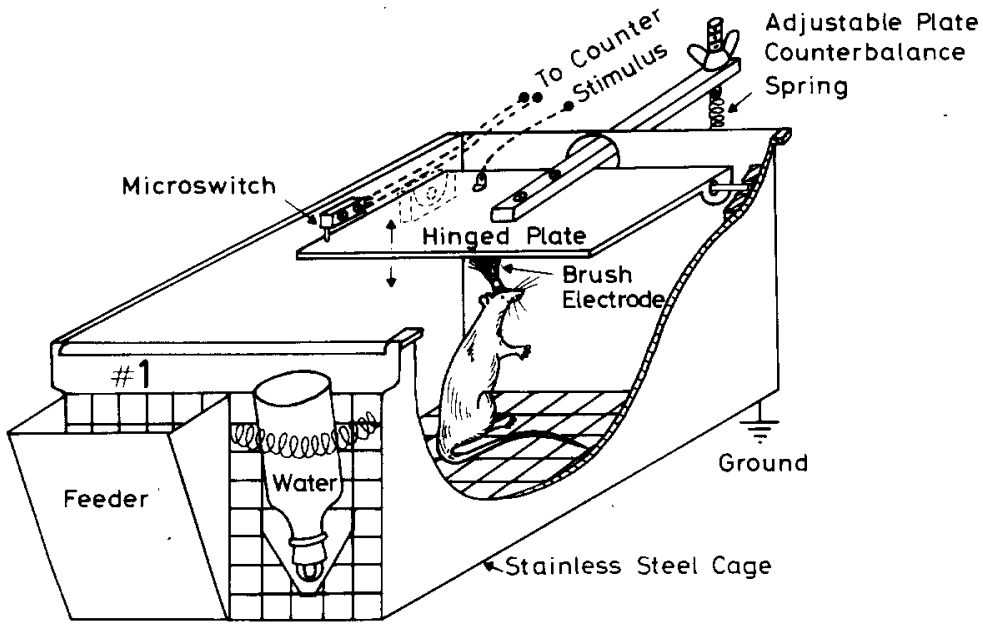

FIG. 1. Schematic illustration of the modified cages for chronic self-stimulation (After Wolf et al., 1973).

stimulation, and self-stimulation without external leads, through the combined contact plate/manipulandum. Stimulation consisted of a 300 millisec train of monopolar 60 cycles per sec sinusoidal current, $45-110 \mu \mathrm{A}$ in intensity. A series of capacitors and resistances were used to assure constant current conditions.

Animals were anaesthetized with sodium pentobarbital (Nembutal, i.p. $50 \mathrm{mg} / \mathrm{kg}$ ) and stereotaxically implanted with unipolar $0.25 \mathrm{~mm}$ diameter nichrome wire electrodes insulated to the tip. Electrodes were aimed at the posterior part of the MFB in the area of the pars compacta of the substantia nigra (SNC) using the coordinate system of Konig \& Klippel (1963) (5.0 mm posterior to Bregma, $1.5 \mathrm{~mm}$ lateral to midline, and $8.0 \mathrm{~mm}$ deep; top of the skull coordinates, zero line at Bregma $=$ A $7200 \mu \mathrm{m})$. The electrodes were attached to a brass brushing which was secured to the skull with stainless steel screws and acrylic dental cement. The implanted animals were placed in the modified cages and the current was turned on. A wooden dowel was used to cause the animals to rear so that the brass brushing contacted the plate and the animals received stimulation. This maneuver was repeated up to 10 times in 2-3 min sessions each and was sufficient to train all the rats. After 3-4 days of initial "shaping" sessions, the animals were allowed 2 additional weeks ad libitum self-stimulation in which rates stabilized. Starting with the third post-operative week, ICS was recorded for 2 consecutive 4-day cycles. The monitoring employed was the same as described for tilt cages and WR. ICS was recorded on an hourly basis, $24 \mathrm{hr} /$ day for the entire period of the experiment. 
On termination of the experiment, all animals were injected with an overdose of sodium pentobarbital and perfused initially with $0.9 \% \mathrm{NaCl}$ followed by a formalin-alcohol-acetic acid fixing solution. The brains were immersed in this solution for at least 2 weeks. From serial $20 \mu \mathrm{m}$ frozen sections of the brains, it was possible to determine the exact locations of the electrodes.

\section{RESULTS}

\section{Wheel running}

The ten animals used in this experiment maintained highly regular 4-day cycles. Figure 2 depicts the mean number of total daily revolutions. Each of the measures was computed for
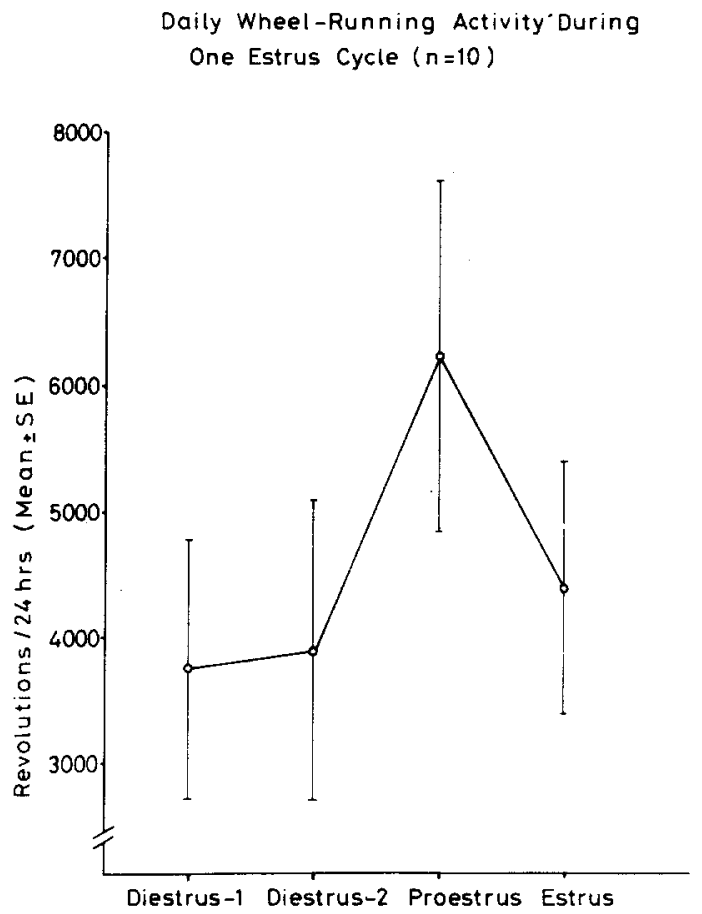

FIG. 2. The mean ( \pm S.E.) number of total 24 -hr revolutions across days of the cycle.

all 10 animals. As shown, the peak in activity when monitored on a $24 \mathrm{hr}$ basis appears to be on the day of proestrus. However, Fig. 3 illustrates the typical activity pattern of WR for these animals on a 3-hourly basis. The actual peak in all animals tested in wheels is somewhere between $2100 \mathrm{hr}$ on the day of proestrus and $0300 \mathrm{hr}$ on the day of estrus. Thus, with this method, it was possible to identify precisely a specific time period, namely the night between proestrus and estrus, as the peak period of WR.

\section{Behavioral taxonomy}

Three categories of behavior occurred with a mean frequency of 3 or more per cycle. These were locomotion, rearing, and grooming. As expected in these nocturnal rodents, a prominent circadian rhythm was observed in all animals with all 3 behaviors. The activity 


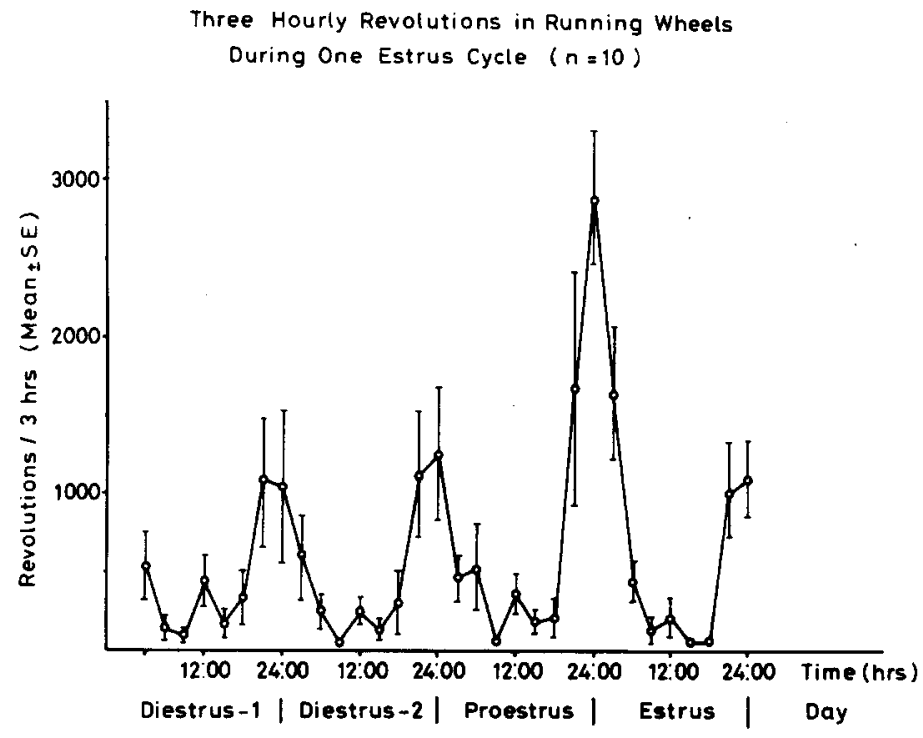

FIG. 3. Typical activity pattern of WR (revolutions per $3 \mathrm{hr}$ ).

was significantly higher $(p<0.001)$ during the dark period. However, there was no significant change in any of the measures across the estrous cycle. Analysis of variance yielded a nonsignificant "condition" (stage in cycle) $\times$ "behavior" interaction $(F=2.93$ for locomotion, 1.32 for rearing, and 0.33 for grooming; $d f=3 / 27$ ). The observational behavioral measures utilized in this experiment do not seem to have a consistent relationship to the estrous cycle.

Tilt cages

The analysis of spectral flatness for locomotor activity as measured in tilt cages over a period of 7 consecutive estrous cycles is summarized in Table I.

TABle I. ANALYSIS OF SPECTRAL FLATNESS FOR LOCOMOTOR ACTIVITY IN TILT CAGES ACROSS SEVEN ESTROUS CYCLES

\begin{tabular}{lcccc}
\hline Subject & $\begin{array}{c}\text { Median spectral } \\
\text { statistic }\end{array}$ & $p<$ & $\begin{array}{c}\text { Pyke's K-S } \\
\text { statistic }\end{array}$ & $p<$ \\
\hline 1 & 1.59 & 0.11 & 0.32 & N.S. \\
2 & 1.16 & 0.25 & 0.28 & N.S. \\
3 & 1.39 & 0.16 & 0.26 & N.S. \\
4 & 0.80 & 0.94 & 0.17 & N.S. \\
5 & 1.37 & 0.17 & 0.31 & N.S. \\
6 & 0.99 & 0.92 & 0.17 & N.S. \\
7 & 2.25 & $0.02^{*}$ & 0.47 & N.S. \\
8 & 1.48 & 0.14 & 0.28 & N.S. \\
9 & 0.24 & 0.80 & 0.48 & N.S. \\
10 & 1.45 & 0.15 & 0.34 & N.S. \\
\hline
\end{tabular}

*Only $12 \%$ accounted for by a 4 -day cycle. 
In using the spectral density analysis and Pyke's $\mathrm{K}-\mathrm{S}$ statistic for each animal, no variability in motor activity over days as measured by tilt cages was demonstrable. Figure 4
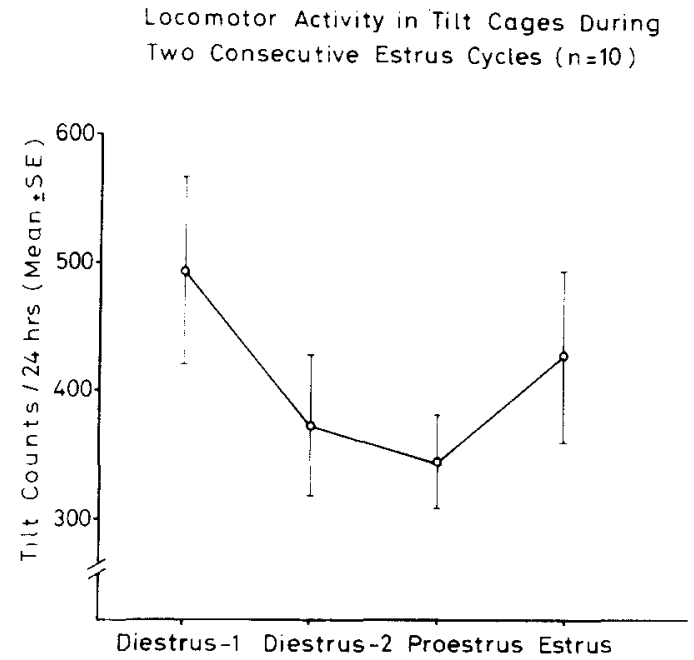

FIG. 4. The mean ( \pm S.E.) 24-hr total counts in tilt cages.

demonstrates this pattern graphically. Nevertheless, the circadian rhythm was prominent and the nocturnal activity was significantly higher $(p<0.001)$ than the activity during the light period. Thus, the apparatus is sensitive enough to show the consistent circadian pattern. When the activity pattern was analyzed separately for the nights only (12-hourly counts during the dark period), an estrous effect became apparent, i.e. a significant increase in tilt activity was observed on the night between estrus and diestrus- $1(p<0.001$; binomial test). The activity measure on a 3-hourly basis (tilt counts per $3 \mathrm{hr}$ ) (Fig. 5) further demonstrates the time span of this late estrus peak. As shown, it occurs on the night between estrus and diestrus-1, and although there seems to be no significant difference between the remaining nights of the cycle, the night between proestrus and estrus tends to show the lowest activity measured. In this experiment tilt cages did not provide a correlative measure for increase in activity on the night between proestrus and estrus.

Intracranial self-stimulation. Recordings in 12 animals have shown that ICS follows exactly the same pattern as WR in relationship to the estrous cycle. Figure 6 shows the mean number of total daily ICS responses for these 12 animals. As with WR, on a 24-hr basis the peak in ICS responses appears to be on proestrus. Figure 7 illustrates the typical ICS response on a 3-hourly basis. The significant increase is again evident on the night between proestrus and estrus and the peak is chronologically the same as the peak in WR (compare with Fig. 3). Figure 8 presents a composite histology of ICS sites for all animals studied in this experiment. All 12 animals maintaining a cyclicity in ICS with a significant peak on the night between proestrus and estrus had their electrodes in or very close to the pars compacta of the substantia nigra (SNC) (see solid circles), also known as area A9 (Hillarp, Fuxe \& Dahlstrom, 1966). Five animals with electrodes outside the SNC did show high but irregular ICS responses despite highly regular 4-day estrous cycles. One animal died shortly after 
surgery (for details see Steiner, 1979). This experiment has shown that ICS of the SNC shows an estrous-linked cyclic response with a peak on the night between proestrus and estrus.

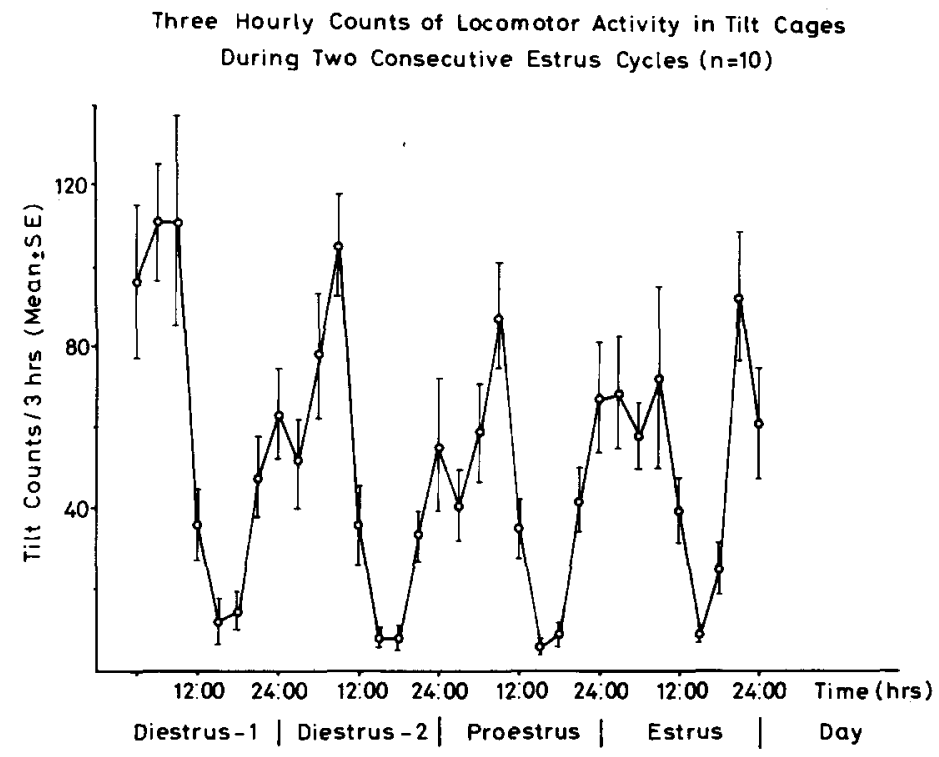

FIG. 5 . The motor activity pattern as measured by tilt cages (counts per $3 \mathrm{hr}$ ).
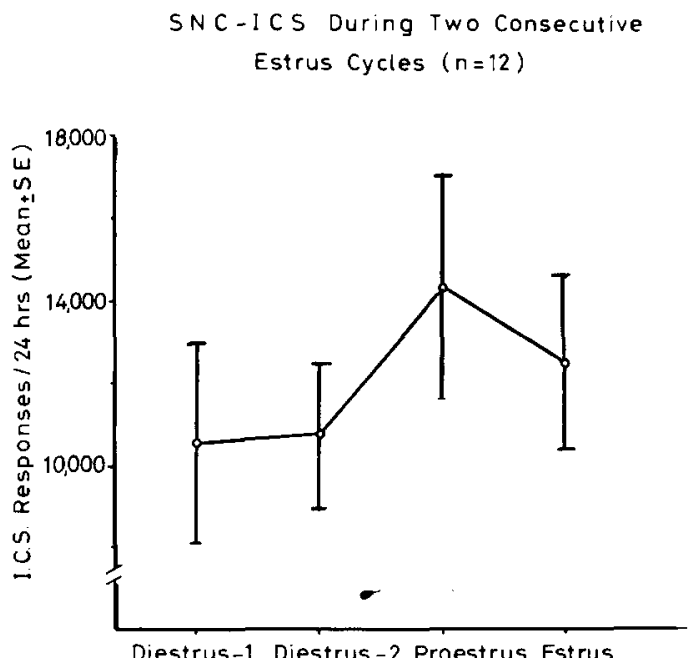

FIG. 6. The mean ( \pm S.E.) number of total daily ICS responses during the estrous cycle. 


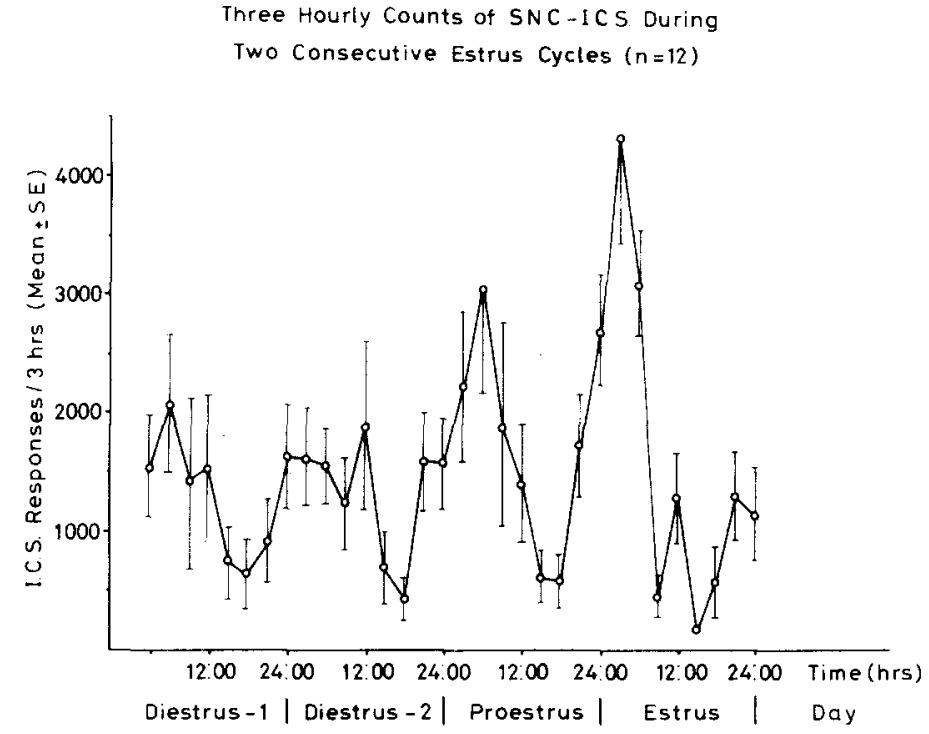

FIG. 7. Typical ICS response on a 3-hourly basis

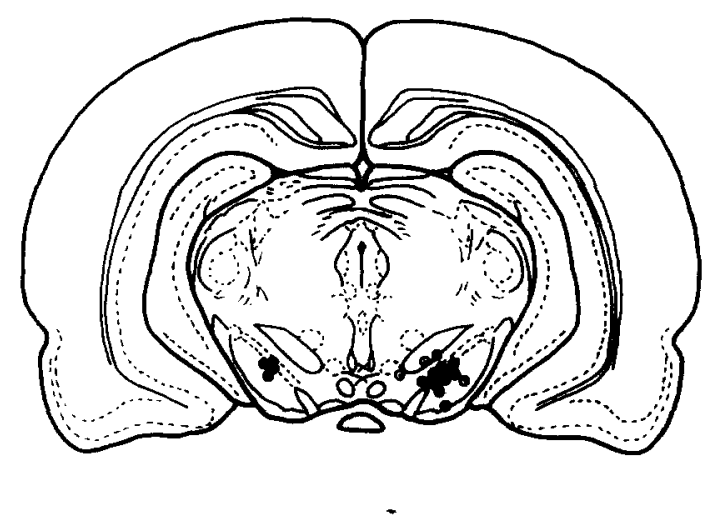

FIG. 8 Composite histology of ICS sites (after Konig \& Klippel, 1963: A 2180 $\mu \mathrm{m}$ ). Electrodes in the pars compacta of the substantia nigra $(\mathbf{O}): 12$ rats show ICS cyclicity with peak on the night between proestrus and estrus. Electrodes outside the SNC $(O)$ : 5 rats show irregular ICS patterns despite highly regular 4-day estrous cycles. 


\section{DISCUSSION}

Of all the behavioral measures tested only WR maintained a highly regular 4-day cyclicity. The temporal structure of WR closely follows the estrous pattern, with a marked peak in activity on the night between proestrus and estrus. From the data on tilt cages, it must be concluded that horizontal cage crossing is different from WR; it may reflect generalized restlessness or irritability and is clearly not a useful response measure for behaviors associated with the night between proestrus and estrus. At the present, it is unclear how much significance needs to be attached to the peak in tilt cage activity observed on the night between estrus and diestrus-1. Locomotion, rearing and grooming do not show an estrous rhythm. These 3 behavioral categories are common naturally occurring behaviors in the rat. They also represent behaviors that under conditions of increased DA sensitivity are enhanced to the point of stereotypes. However, none of these behaviors or conditions seems to have a cyclicity which is comparable to the changes observed on the night between proestrus and estrus. The difference in what is measured by the running wheels as opposed to the other instruments seems to be at least suggestive of a more complex underlying mechanism. WR is energy consuming, it does not habituate, it is sensitive to changes in metabolic state, to circadian rhythms, and to the estrous cycle. In addition to the motor activity that WR measures, it represents a highly motivated behavior (Rosenquist \& Hoebel, 1968). WR does not belong to the natural repertoire of the animals, and in this experiment, the access to the wheels was voluntary. There is some evidence, however, that goal-oriented running per se, as opposed to nonspecific locomotion, is linked to sexually motivated behavior (Meyerson \& Lindstrom, 1973) and as such has a biological meaning for the survival of the species. One is tempted to speculate that at least in part, WR has also a pleasurable aspect and serves a hedonic function (Collier, Hirsch \& Kanarek, 1977).

The SNC-ICS results (to our knowledge never reported before in the literature with this design) have shown that ICS follows exactly the same pattern as WR in relation to the estrous cycle, with a marked peak in ICS activity on the night between proestrus and estrus. This ICS behavioral cyclicity is dependent on the electrical stimulation and can not be accounted for by the non-specific act of rearing (Steiner, 1979). A specificity of a locus or a pathway involved in the ICS-estrus linked cyclic response has been identified. The pars compacta of the substantia nigra is known to contain dopamine neurons. If the DA systems are considered to be part of a primary reinforcer-incentive-motor system circuitry (Iversen, 1977), it is quite possible that the function of this system changes during the estrous cycle. The specificity of the SNC has been established further by records obtained from the 5 animals with electrodes outside the SNC. These animals showed high but irregular ICS counts, which were not related to the estrous cycle despite the fact that these animals maintained regular 4-day cycles. Thus, whatever the changes might be that cause a change in ICS on the night between proestrus and estrus, they are not generalized changes in brain sensitivity. Whether or not the changes are specific to the SNC still needs to be determined. Further studies of additional ICS sites across the estrous cycle utilizing the specific design described here are needed.

The specific increases in motivated behaviors as expressed by WR, ICS and the seeking of sexual behavior follow the same rhythms, are synchronized and all peak on the night between proestrus and estrus. These behaviors can be dissociated from nonspecific non-goal- 
oriented, general motor activities which by contrast do not show any relationship to the cycle. The results so far imply a neuroendocrine linkage between estrous-related fluctuations and catecholamine mediated changes in motivated behaviors.

\section{REFERENCES}

Baumeister, A.. Hawkins, W. F. \& Cromwell, R. L. (1964) Need states and activity level. Psw hol. Bull. 61, 438453.

Bolles, R. C. (1963) A failure to find evidence of the estrous cycle in the rat's activity level. Psychol. Rep. 12,530

Butcher, R. L., Collins, W. E. \& Fugo, N. W. (1974) Plasma concentration of LH, FSH, prolactin, progesterone and estradiol- 17 beta throughout the 4-day estrous cycle of the rat. Endocrinol. 94, $1704-1708$.

Collier, G., Hirsch, E. \& Kanarek, R. (1977) The operant revisited. In Handbook of Operant Bohaviour W. K. Honig and J. E. R. Staddon (Eds.), pp. 28-52. Prentice-Hall, Englewood Cliffs. New Jersey.

Dixon, W. J. (1968) Biomedical Computer Programs. University of California Press, Berkeley.

Drewett, R. F. \& HERBERG, L. J. (1975) Hypothalamic self-stimulation in the female rat: effects of oestrus and food deprivation. Physiol. Behav. 14, 285 289.

Finger, F. W. (1969) Estrus and general activity in the rat. J. Comp. Physiol. Psychol. 68, 461 466.

Fox, D. J. \& GulRE, K. E. (1976) Documentation for MID.AS. 3rd Edition. The University of Michigan. Statistical Research Laboratory, Ann Arbor, Michigan.

Hillarp, N. A., Fuxe, K. \& Dahistrom, A. (1966) Demonstration and mapping of central neurons containing dopamine, noradrenaline, and 5-hydroxytryptamine and their reactions to psychopharmaca. Pharmac'ol. Re' 18. $727-741$.

Hitt, J. C. \& Glrall, A. A. (1969) Effect of brain stimulation on estrous activity cycles. Psichol. Rep. 24, 59 68.

Hodos. W. \& Valenstein, E. S. (1960) Motivational variables affecting the rate of behavior maintained by intracranial stimulation. J. Comp. Phwsiol. Psichol. 53,502 508 .

IVERSEN. S. D. (1977) Striatal function and stereotyped behaviour. In Pstchobiology of the Striatum. A. R. Coxs ('t al., (Eds.), pp. 99-118 Elsevier/North-Holland Biomedical Press, Amsterdam.

KAGAN, J. \& BERKUN. M. (1954) The reward value of running activity. I. Comp. Physiol. Psychol. 47, 108.

Konig. J. F. R. \& K LiPPel, R. A. (1963) The Rat Brain: A Stereotaxic Allas of the Forebrain and Lower Parts of the Brain Stem. Robert E. Krieger Co., Huntington, New York.

Lenzer, I. I. (1971) Food deprivation, estrogen levels and self-stimulation in the female rat. Psychol. Rep. 29, 655 665 .

LIVESEY, P. J., Egger, G. J. \& Meyer. P. N. (1972) Wheel running, a rewarding activity for the rat or response to increased drive following deprivation. Austral. J. Psychol. 24, 45 53.

Meyerson. B. J. \& Lindstrom. L. H. (1973) Sexual motivation in the female rat: a methodological study applied to the investigation of the effect of estradiol benzoate. Acta Physiol. Scand. Suppl. 389.

Meyerson, B. J., Wilkins, S. E. \& SAWYER, C. H. (1969) Effects of estrogen on self-stimulation behavior in ovariectomized rats. Physiol. Behav. 4, 291-296.

Moss, R. L. (1968) Changes in bar-press duration accompanying the estrous cycle. $J$. (omp. Phisiol. Psichol. 66, $460-466$

Prescott, R. G. W. (1966) Estrous cycle in the rat: effects on self-stimulation behavior. Scicnce 152, 796.797.

Rosenquist. A. C. \& Hoebfi., B. G. (1968) Wheel running elicited by electrical stimulation of the brain. Phisiol. Behar. 3, 563-566.

Scott, T. R. Jr. \& Hoebel, B. G. (1966) Effect of sex hormones on lateral hypothalamic self-stimulation and feeding. Amer. Zool. 6, 537.

STEINER, M. (1979) Motivated behavior and the estrous cycle of the rat. Ph. D. Dissertation. University of Michlyan.

WANG, G. H. (1923) The relation between "spontaneous" activity and oestrous cycle in the white rat. Comp. P.ivd Monogr. 2, 1-27.

WARNer, L. H. (1927) A study of sex behavior in the white rat by means of the obstruction method. (onp. Psichol. Monogr. 4, I 58 .

Wolf, G., DiCara, L. V. \& Simpson, W. (1973) The contact method: a simple technique for electrical selfstimulation without external leads. Physiol. Behar. 11, 721 723.

Zarrow, M. X., Yochim, J. M. \& MCCARThY, J. L. (Eds.) (1964) Experimental Endocrinologl". A Sourcehook of Basic Techniques, pp. 36-39. Academic Press, New York. 